\title{
Metamaterial composite bandpass filter with an ultra-broadband rejection bandwidth of up to 240 terahertz
}

Strikwerda, Andrew; Zalkovskij, Maksim; Lorenzen, Dennis Lund; Krabbe, Alexander; Lavrinenko, Andrei; Jepsen, Peter Uhd

Published in:

Applied Physics Letters

Link to article, DOI:

$10.1063 / 1.4875795$

Publication date:

2014

Document Version

Publisher's PDF, also known as Version of record

Link back to DTU Orbit

Citation $(A P A)$ :

Strikwerda, A., Zalkovskij, M., Lorenzen, D. L., Krabbe, A., Lavrinenko, A., \& Jepsen, P. U. (2014). Metamaterial composite bandpass filter with an ultra-broadband rejection bandwidth of up to 240 terahertz. Applied Physics Letters, 104(19), [191103]. https://doi.org/10.1063/1.4875795

\section{General rights}

Copyright and moral rights for the publications made accessible in the public portal are retained by the authors and/or other copyright owners and it is a condition of accessing publications that users recognise and abide by the legal requirements associated with these rights.

- Users may download and print one copy of any publication from the public portal for the purpose of private study or research.

- You may not further distribute the material or use it for any profit-making activity or commercial gain

- You may freely distribute the URL identifying the publication in the public portal 


\section{AIP Appled phy \\ Letters}

\section{Metamaterial composite bandpass filter with an ultra-broadband rejection bandwidth of up to 240 terahertz}

Andrew C. Strikwerda, Maksim Zalkovskij, Dennis Lund Lorenzen, Alexander Krabbe, Andrei V. Lavrinenko, and

Peter Und Jepsen

Citation: Applied Physics Letters 104, 191103 (2014); doi: 10.1063/1.4875795

View online: http://dx.doi.org/10.1063/1.4875795

View Table of Contents: http://scitation.aip.org/content/aip/journal/apl/104/19?ver=pdfcov

Published by the AIP Publishing

\section{Articles you may be interested in}

Orthogonally twisted planar concentric split ring resonators towards strong near field coupled terahertz metamaterials

Appl. Phys. Lett. 104, 101105 (2014); 10.1063/1.4868122

Design and fabrication of a high transmissivity metal-dielectric ultraviolet band-pass filter

Appl. Phys. Lett. 102, 213105 (2013); 10.1063/1.4807925

Active terahertz quantum-cascade composite right/left-handed metamaterial

Appl. Phys. Lett. 102, 021103 (2013); 10.1063/1.4775666

An ultrabroad terahertz bandpass filter based on multiple-resonance excitation of a composite metamaterial Appl. Phys. Lett. 99, 191909 (2011); 10.1063/1.3660273

Bandpass Filter Based On Parallel Cascaded Multiple Microring Resonators

AIP Conf. Proc. 709, 417 (2004); 10.1063/1.1764036

\section{NEW! Asylum Research MFP-3D Infinity ${ }^{\text {TM }}$ AFM}

Unmatched Performance, Versatility and Support

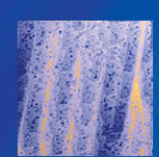

Comprehensive tools for nanomechanics
Stunning high performance
Simpler than ever to GetStarted ${ }^{\mathrm{TM}}$
The Business of Science ${ }^{\mathrm{e}}$

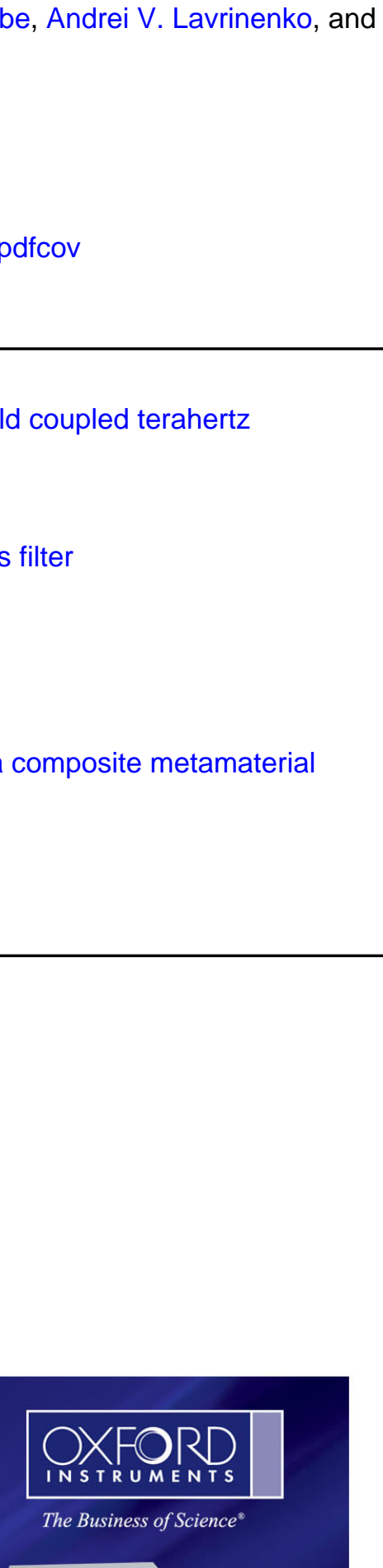

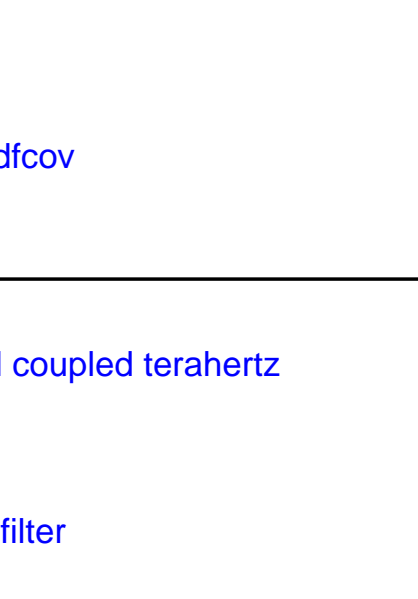

. 


\title{
Metamaterial composite bandpass filter with an ultra-broadband rejection bandwidth of up to 240 terahertz
}

\author{
Andrew C. Strikwerda, ${ }^{a}$ Maksim Zalkovskij, Dennis Lund Lorenzen, Alexander Krabbe, \\ Andrei V. Lavrinenko, and Peter Und Jepsen \\ DTU Fotonik-Department of Photonics Engineering, Technical University of Denmark, DK-2800 Kongens \\ Lyngby, Denmark
}

(Received 21 February 2014; accepted 28 April 2014; published online 12 May 2014)

\begin{abstract}
We present a metamaterial, consisting of a cross structure and a metal mesh filter, that forms a composite with greater functional bandwidth than any terahertz ( $\mathrm{THz}$ ) metamaterial to date. Metamaterials traditionally have a narrow usable bandwidth that is much smaller than common $\mathrm{THz}$ sources, such as photoconductive antennas and difference frequency generation. The composite structure shown here expands the usable bandwidth to exceed that of current $\mathrm{THz}$ sources. To highlight the applicability of this combination, we demonstrate a series of bandpass filters with only a single pass band, with a central frequency $\left(f_{0}\right)$ that is scalable from $0.86-8.51$ $\mathrm{THz}$, that highly extinguishes other frequencies up to $>240 \mathrm{THz}$. The performance of these filters is demonstrated in experiment, using both air biased coherent detection and a Fourier transform infrared spectrometer (FTIR), as well as in simulation. We present equations - and discuss their scaling laws - which detail the $f_{0}$ and full width at half max $(\Delta f)$ of the pass band, as well as the required geometric dimensions for their fabrication using standard UV photolithography and easily achievable fabrication linewidths. With these equations, the geometric parameters and $\Delta f$ for a desired frequency can be quickly calculated. Using these bandpass filters as a proof of principle, we believe that this metamaterial composite provides the key for ultra-broadband metamaterial design. (C) 2014 AIP Publishing LLC. [http://dx.doi.org/10.1063/1.4875795]
\end{abstract}

Since their introduction nearly fifteen years ago, metamaterials ${ }^{1-3}$ have provided a unique design paradigm. The concept of individually tuning the permittivity and permeability—or index and impedance, if you prefer-excited the community with a negative index of refraction, ${ }^{2,3}$ imaging past the diffraction limit, ${ }^{4}$ and transformation optics. ${ }^{5}$ Those lofty motivations have gradually devolved into more traditional, but imminently practical, applications such as dielectric sensing, ${ }^{6}$ polarization control, ${ }^{7,8}$ modulation, ${ }^{9}$ absorption, ${ }^{10}$ and detection. ${ }^{11,12}$ However, the initial motivation of designing the permittivity and permeability often limits the study of metamaterials to a small bandwidth around their design frequency. This bandwidth limitation is due to several factors such as the resonant nature of most metamaterials, the appearance of unwanted higher order modes, and the prevalence of the effective medium approximation where metamaterials are only considered to have a "designed" permittivity and permeability in the deep sub-wavelength regime. ${ }^{13}$ While this behavior can be easily integrated with narrowband applications, the two most common methods of terahertz (THz) generation, photoconductive antennas, ${ }^{14}$ and nonlinear generation in either crystals ${ }^{15}$ or air plasma, ${ }^{16}$ both yield broad spectra that suggest there is an unfulfilled need for broadband $\mathrm{THz}$ components.

Here, we reconcile the disconnect between traditional broadband sources and narrowband metamaterial components by demonstrating that a metamaterial composite can have an ultra-broadband usable range. As a proof of principle of this ultra-broadband concept, we have made a series of

\footnotetext{
${ }^{\text {a) }}$ Author to whom correspondence should be addressed. Electronic mail: astr@fotonik.dtu.dk.
}

bandpass filters that display the largest usable bandwidth of any THz metamaterial device to date. The filters have a single pass band, with a central frequency scalable between 0.86-8.51 THz, while severely attenuating all other frequency components to $>240 \mathrm{THz}$. These filters, which operate due to a trapped mode excitation, were originally introduced to the $\mathrm{THz}$ regime by Paul et al., from 0-2.5 THz. ${ }^{17}$ We have customized their structure and expanded the bandwidth by almost two orders of magnitude.

To clearly identify the two constituent components in the metamaterial composite, a cross element, and a metal mesh, ${ }^{18,19}$ we present an optical picture of a $2 \times 2$ array of unit cells in Figure 1(a). The unit cell in the bottom right, outlined in black, clearly identifies the cross component. However, this unit cell choice suggests that the cross is placed inside of its own, slightly larger, Babinet complement. ${ }^{20}$ If the unit cell is translated by $(-1 / 2,1 / 2) \times \mathrm{P}$, to the grey outlined unit cell, a different structure is suggested. In this new unit cell, if the cross is ignored, it can be seen that the Babinet complement is also a metal mesh filter.

The sample dimensions in the figure-except for $\varepsilon$, which was held constant at $1.5 \mu \mathrm{m}$ - are all scaled by a single dimensionless scaling parameter $(\sigma)$ according to the following equations: $\mathrm{L}=7.09 \times \sigma ; \mathrm{W}=4.5 \times \sigma ; \mathrm{P}=10.03 \times \sigma$; where all dimensions are in microns. The cross element will always have a length and width of $\mathrm{L}-2 \times \varepsilon$ and $\mathrm{W}-2 \times \varepsilon$, respectively. The constant value of $\varepsilon$ provides an easily achievable minimum linewidth for fabrication using UV photolithography, and the samples are polarization insensitive due to their four fold symmetry. We fabricated two different styles of samples: single- and double-sided. Both styles have the exact same metamaterial pattern, except that the double-sided 


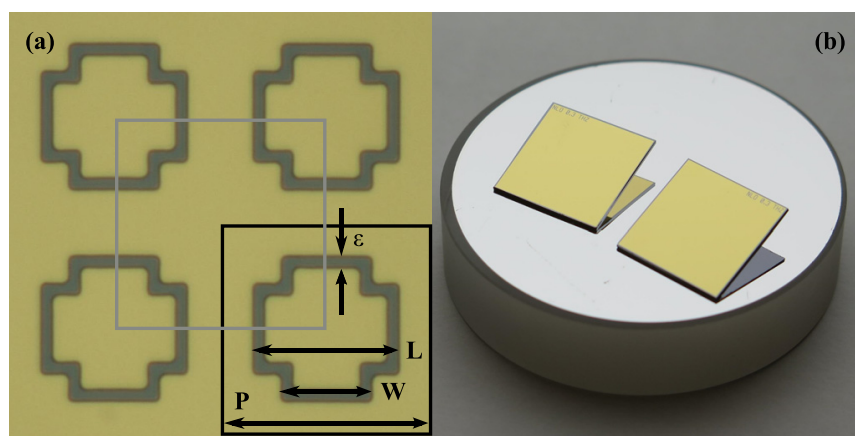

FIG. 1. Optical pictures of fabricated samples. (a) A $2 \times 2$ picture of the composite filter. The two different choices of unit cells, represented by the black and grey outlines, help identify the cross and metal mesh, respectively. The minimum linewidth $(\varepsilon)$ is held constant at $1.5 \mu \mathrm{m}$ for every sample. $\mathrm{L}$, $\mathrm{W}$, and $\mathrm{P}$ all scale with the dimensionless parameter, $\sigma$, as described in the text and Table I. The layer is entirely gold except for the small linewidth defined by $\varepsilon$, which is HR-Si. (b) A single-sided sample (right) and a double-sided sample (left). The mirror clearly shows the double-sided sample's second metallization layer on the backside of the HR-Si substrate. The cross section of the single-sided sample is gold/HR-Si, while the doublesided sample is gold/HR-Si/gold.

structure has the pattern on both sides of the $525 \mu \mathrm{m}$ thick high resistivity silicon (HR-Si) substrate as shown in Fig. 1(b). The dimensions, $\sigma$ values, central frequency $\left(f_{o}\right)$, and full width at half $\max (\Delta f)$ of the filters studied are presented in Table I. For reference, these dimensions were originally chosen so that the higher order modes of the cross geometry would couple to lattice modes to eliminate unwanted transmission modes, although this ended up being unnecessary as discussed later.

To highlight the effect of the two constituent components in the structure, we present the simulated ${ }^{21}$ transmission curves of the cross, metal mesh, and full composite structure in Figure 2. The metal mesh is easily described as a combination of inductive and capacitive meshes. ${ }^{22}$ A capacitive mesh, which is simply a two dimensional array of metallic squares, works as a low pass filter. The complementary structure, called an inductive mesh, is a wire grid that functions as a high pass filter. Their combination, a metal mesh filter as shown here, has a pass band located at $\mathrm{c} /\left(\mathrm{n}_{\mathrm{Si}} \times \mathrm{P}\right)$, where $\mathrm{c}$ is the speed of light, $\mathrm{n}_{\mathrm{Si}}$ is the refractive index of the silicon substrate, and $\mathrm{P}$ is the unit cell size. ${ }^{18}$ At higher frequencies, the transmission through the mesh can be closely

TABLE I. The physical dimensions of the fabricated samples, their simulated central frequency $\left(f_{0}\right)$, and full width half max for single- $\left(\Delta f_{1}\right)$ and double-sided $\left(\Delta f_{2}\right)$ styles. $\sigma$ is dimensionless, $\mathrm{L}, \mathrm{W}$, and $\mathrm{P}$ are in microns, and all frequencies are in THz.

\begin{tabular}{lcccccc}
\hline \hline$\sigma$ & $\mathrm{L}$ & $\mathrm{W}$ & $\mathrm{P}$ & $f_{0}$ & $\Delta f_{1}$ & $\Delta f_{2}$ \\
\hline 1.0 & 7.1 & 4.5 & 10.0 & 8.51 & 5.76 & 2.94 \\
1.125 & 8.0 & 5.1 & 11.3 & 7.24 & 4.77 & 2.57 \\
1.25 & 8.9 & 5.6 & 12.5 & 6.25 & 4.10 & 2.30 \\
1.5 & 10.6 & 6.8 & 15.0 & 4.89 & 3.19 & 1.93 \\
1.75 & 12.4 & 7.9 & 17.6 & 3.97 & 2.51 & 1.58 \\
2.0 & 14.2 & 9.0 & 20.1 & 3.33 & 2.02 & 1.30 \\
2.5 & 17.7 & 11.3 & 25.1 & 2.53 & 1.58 & 0.97 \\
3.125 & 22.2 & 14.1 & 31.3 & 1.91 & 1.19 & 0.72 \\
3.75 & 26.6 & 16.9 & 37.6 & 1.54 & 0.95 & 0.56 \\
6.25 & 44.3 & 28.1 & 62.7 & 0.86 & 0.49 & 0.29 \\
\hline \hline
\end{tabular}

modeled by taking the geometric ratio of metal area to unit cell area multiplied by the Fresnel transmission coefficient of silicon. In Figure 2, this ratio for each component is represented by the dotted horizontal line. When the cross element is added to the metal mesh, both the pass band and the high frequency content are substantially modified. The pass band is red shifted, resulting in the previously mentioned trapped mode excitation, ${ }^{17}$ and the high frequency content is reduced significantly. It is worth noting that even though the high frequency transmission of both the mesh filter and cross can be closely modeled by their geometric ratios, the composite structure shows an even greater extinction.

The dashed magenta line in Figure 2 is the simulated transmission of a single metamaterial element where the periodic boundary conditions have been replaced by perfectly matched layers. This allows us to separate the behavior of a single element from the behavior of the periodic lattice. The single element transmission roughly follows the transmission of the full structure with two major differences. First, the transmission through the periodic lattice is larger than through a single element, which can be explained through the coherent superposition of multiple elements. This will result in strongly focused scattering in the forward direction, as opposed to the larger angular distribution of a single element. The second difference between the curves is the appearance of sharp modes on the transmission of the full structure. These lines can all be attributed to lattice modes, and are calculated using $f_{j, k}=c_{n} \sqrt{j^{2}+k^{2}} / \mathrm{P}$ where $\mathrm{c}_{\mathrm{n}}$ can be the speed of light in either air or silicon and $\mathrm{j}$ and $\mathrm{k}$ are integers representing the mode order. The modes present in this figure go up to $f_{2,0}(29.85 \mathrm{THz})$ for the air side of the filter and $f_{6,3}(29.3 \mathrm{THz})$ for the silicon side. There are also local maximums in the single element transmission near $10.2 \mathrm{THz}$ and $22.5 \mathrm{THz}$. An examination of the current patterns at the gold-silicon interface suggests that these are higher order

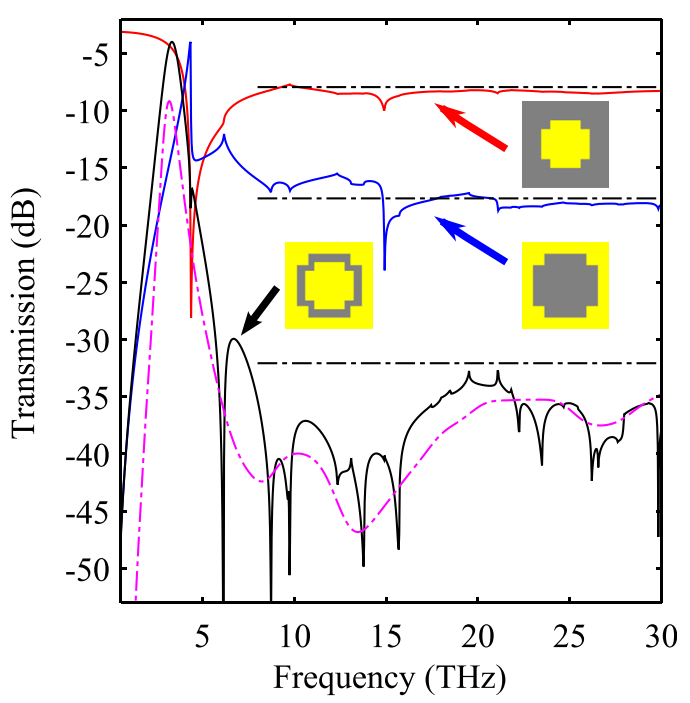

FIG. 2. The simulated transmission spectrum of a double-sided metamaterial and metal mesh filter composite. The dimensions correspond to the $\sigma=2$ structure in Table I. The red line is the transmission of the cross element without the metal mesh, the blue line is the metal mesh filter without the cross, the black line is the full metamaterial composite, and the dashed magenta line is a single element instead of a full periodic array. The dotted lines represent the geometric ratio of metallization area divided by unit cell area times the Fresnel transmission coefficient of the HR-Si wafer. 
modes of the cross element ( $\mathrm{n}=3$ and $\mathrm{n}=5$, respectively), but their highly oscillatory current distribution likely explains their weak coupling to the incident plane wave, as well as the absence of any other transmission bands.

To verify these simulated results, the fabricated samples were measured with THz-time domain spectroscopy using a two-color air plasma for generation ${ }^{23}$ and air biased coherent detection. ${ }^{24}$ This system does have a non-traditional BesselGauss beam profile, ${ }^{25}$ but this beam profile is simply a superposition of Gaussian beams and is subsequently irrelevant to the filter performance. The optical pulse length used to generate the air plasma and $\mathrm{THz}$ beam was $35 \mathrm{fs}$, yielding an anticipated bandwidth of $\sim 1 / 35 \mathrm{fs}=28.6 \mathrm{THz}$ which is approximately that achieved in our reference measurements. Further details of the experimental system can be found elsewhere. ${ }^{26}$ The samples were also measured in a Fourier transform infrared spectrometer (FTIR) to examine their high frequency extinction up to $240 \mathrm{THz}(1.25 \mu \mathrm{m} / 0.99 \mathrm{eV})$.

An aggregate comparison between simulation and experiment for all samples is shown in Figure 3. The two plots compare $f_{0}$ and $\Delta f$ versus $\sigma$ for both the single- and double-sided samples. Fits to the data were conducted using a power law and the simulated values in Table I. The resulting equations are $f_{0}=8.22 \times \sigma^{-1.42}+0.28 ; \Delta f_{l}=5.51$ $\times \sigma^{-1.56}+0.22$; and $\Delta f_{2}=3.16 \times \sigma^{-1.05}-0.21$, where $\Delta f_{1}$ is for the single-sided sample and $\Delta f_{2}$ is for the double-sided. For fit details, see Ref. 27. Note that both the single- and double-sided samples share the same resonance frequency, because, due to the relative thickness of the HR-Si substrate, there is no coupling between these two layers and they can (a) $f_{0}$

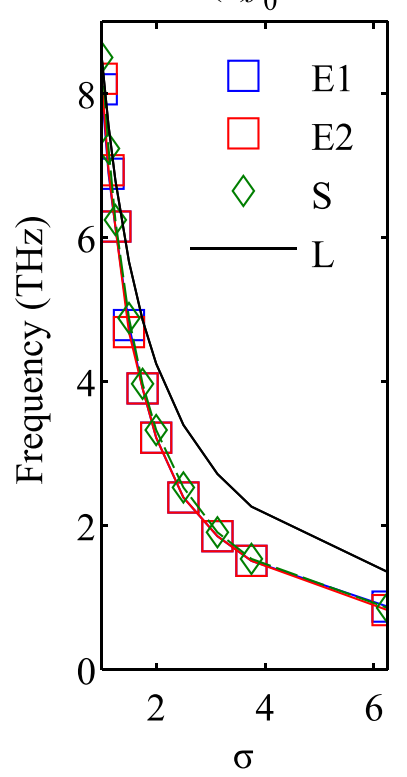

(b) $\Delta f$

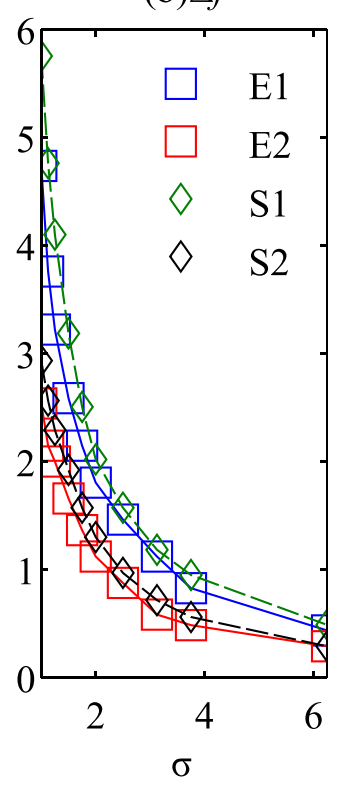

FIG. 3. (a) The aggregate agreement between $f_{0}$ in simulation and experiment. Not all data are visible because of the virtual overlap between simulation and experiment. E1/E2 refer to experimental values for single- and double-sided samples and $\mathrm{S}$ refers to simulation. To highlight the effect of the constant $\varepsilon$ in all structures, the solid black line (L) represents a linear scaling of $f_{O}$ vs $\sigma$, where $f_{O}$ is for the $\sigma=1$ simulation. (b) Comparison of the single- and double-sided $\Delta f$ for experimental (E) and simulated (S) results. Note that the lines shown are visual aids connecting adjacent data points and not fits. The simulated data points for both figures are presented in Table I, and a power law fit is given in the text. be treated independently at the band pass frequencies. ${ }^{28}$ The double-sided structures show a reduced $\Delta f$ due to transmission through two filters, demonstrating that multiple filters can be stacked to achieve an even narrower bandwidth, as required. It is our hope that these design equations can be used to quickly fabricate bandpass filters for any frequency in this range. Simply calculate $\sigma$ for the desired $f_{0}$, use the geometric equations to determine $\mathrm{L}, \mathrm{W}$, and $\mathrm{P}$, and then calculate $\Delta f_{1}$ and $\Delta f_{2}$ for the subsequent filters. Since $\mathrm{L}, \mathrm{W}$, and $\mathrm{P}$ are linear with $\sigma$, they also have a nonlinear relationship with frequency and the same scaling behavior as $\sigma$. They decrease monotonically from $\mathrm{L}=0.20 \times \lambda_{0}, \mathrm{~W}=0.13 \times \lambda_{0}$, and $\mathrm{P}=0.28 \times \lambda_{0}$ for $\sigma=1$ to $\mathrm{L}=0.13 \times \lambda_{0}, \mathrm{~W}=0.08 \times$ $\lambda_{0}$, and $\mathrm{P}=0.18 \times \lambda_{0}$ for $\sigma=6.25$, where $\lambda_{0}$ is the free space wavelength at $f_{0}$.

Metamaterials are well-known to be scale invariant, yet our scaling equations are clearly not linear with sample size. This scale invariance is broken by the constant value of $\varepsilon$, which results in increased coupling in the trapped mode excitation with increasing $\sigma$ and causes a red shift in $f_{0}$. As a visual aid, Figure 3(a) has a line that is $f_{0}$ of the $\sigma=1$ filter scaled linearly with $\sigma$. The deviation of the results from this line demonstrates the aforementioned red shift vs $\sigma$.

We can model this increased coupling by assuming that the capacitance of the metamaterial composite is dominated by the capacitive coupling between the cross element and the metal mesh filter. We begin by describing the filter as a resonant element, where $f_{0} \sim 1 / \sqrt{L C}$ and $L$ and $C$ are the total inductance and capacitance of the metamaterial, respectively. Next, we assume that the capacitive coupling between the cross element and the metal mesh filter can be approximated as a parallel plate capacitor with capacitance $C \sim$ area/distance, and this contribution dominates the total capacitance of the structure. Making this substitution for $C$, we see that $f_{0} \sim N \sqrt{\varepsilon}$, where $\varepsilon$ is the distance between the cross element and the metal filter, and every other dependency has been lumped into the unknown variable $N$. The scale invariance of Maxwell's equations tell us that if we assume $\varepsilon=1.5 \times \sigma$, then every dimension would scale linearly and the resonance frequency would match the linear approximation plotted in Figure 3(a). This means that $f_{0} \sim N \sqrt{1.5 \times \sigma} \sim \sigma^{-1}$, and therefore, $N \sim \sigma^{-3 / 2}$. If we instead hold $\varepsilon$ constant, as we do in our metamaterial samples, we have $f_{0} \sim N \sqrt{\varepsilon} \sim N$ $\sim \sigma^{-3 / 2}$. This yields an exponent of -1.5 , which agrees closely with our fitted value of -1.42 .

The scaling of $\Delta f_{l}$ can be described in a similar manner. If we assume that the majority of the energy stored in the filter is in the electric field of the previously mentioned "capacitor," we can use $Q \sim 1 / f_{0} R C$ for a capacitive element. Again using our substitutions for $f_{0}$ and $\mathrm{C}$, we have $Q \sim 1 / f_{0} C \sim 1 / \sqrt{C} \sim \sqrt{\varepsilon}$ which is constant. Since we also know that $Q \sim f_{0} / \Delta f$, a constant $Q$ implies that $f_{0}$ and $\Delta f$ scale identically, and, therefore, $\Delta f_{1} \sim \sigma^{-3 / 2}$, which is again close to the fitted value of -1.56 . While this simple argument ignores any changes due to fringing fields, surface capacitance, ${ }^{29}$ and inductance, the agreement with the fitted scaling equations suggests that this simple capacitive coupling argument captures the essence of the physics at play.

We have also examined $\Delta f$ for transmission through up to six filters and have, for the sake of design convenience, 
(a) Experimental

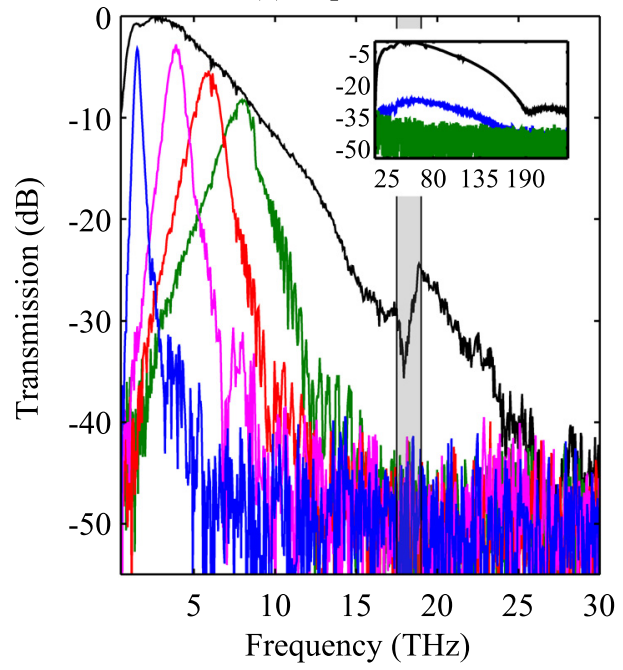

(b) Simulation

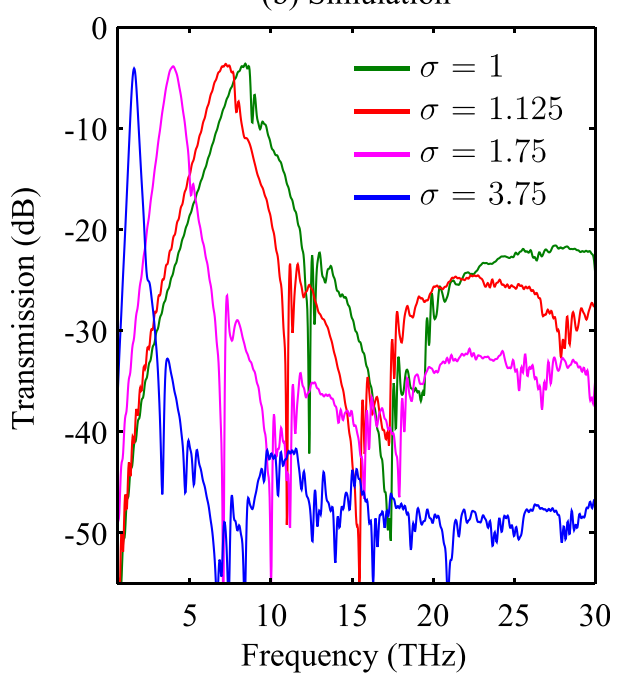

FIG. 4. (a) Several spectra from experimental ABCD and FTIR (inset) measurements and (b) simulation. For the sake of clarity, only a subset of the structures is displayed. There is one major difference between these figures. The simulated spectra are normalized and, therefore, represent the frequency dependent transfer function. The experimental spectra are not normalized, and therefore, the reference spectrum used to measure them (shown in black) is also included. This is because the dynamic range of the experimental measurement is limited by the difference between the reference signal and the noise floor. ${ }^{31}$ For example, the reference is $\sim-30 \mathrm{~dB}$ at $20 \mathrm{THz}$, while the noise floor is $-50 \mathrm{~dB}$. This $20 \mathrm{~dB}$ dynamic range is insufficient to capture the full extinction of the filters at this frequency, as demonstrated in (b). Also note that we include only one of several reference scans taken throughout the measurements- the peak transmission for each sample was $80 \%-90 \%$ relative to its own reference. The highlighted grey window in the experimental spectrum identifies a phonon absorption ${ }^{32}$ in a HR-Si wafer that is intrinsic to the THz beam path. (inset of (a)) FTIR measurements of $\sigma=3.75$ single- (blue) and double-sided (green) samples and the reference spectrum (black), demonstrating that the extinction continues up to $240 \mathrm{THz}(1.25 \mu \mathrm{m} / 0.99 \mathrm{eV})$.

included the fitted equation for $\Delta f_{2}$ listed previously. However, we hasten to add that we do not attach any physical significance to the scaling behavior of this equation. This is because the changes in capacitive coupling strength due to constant $\varepsilon$ result in changes to the transmitted lineshape between the various $\sigma$ samples. When squared, cubed, etc., these different lineshapes all display reduced $\Delta f$, but not in a consistently meaningful way. As an example of this lineshape difference, an asymmetry can be seen in the $\sigma=1$ pass band in Figure 4(a) which is due to a slight decoupling of the trapped mode excitation.

In Fig. 4, we demonstrate the broadband agreement between the experimental and simulated results. For clarity, only a subset of the samples is shown in this figure. In the inset of Fig. 4(a), we present the FTIR spectra for the $\sigma=3.75$ sample, which goes from $10.4-240 \mathrm{THz}$. The relatively featureless transmission displayed is representative of all the samples. In particular, it is worth pointing out that the single-sided sample (blue) is slowly approaching the reference (black) with increasing frequency from $-29 \mathrm{~dB}$ at 30 $\mathrm{THz}$ to $-23 \mathrm{~dB}$ at $160 \mathrm{THz}$ when the signal approaches the noise floor. Our simple geometric extinction argument, which generated the vertical dashed lines in Fig. 2, predicts a high frequency extinction of $-22.5 \mathrm{~dB}$. More generally, this simple calculation matches the data within a few $\mathrm{dB}$ for all samples.

The choice of constant $\varepsilon$ has an impact on both the high and low frequency behavior. While not shown here, the simulated peak transmission through a double-sided 0.23 $\mathrm{THz}$ filter $(\sigma=20)$ is only $0.4(-8 \mathrm{~dB})$. This decrease is understandable, given that $\varepsilon$ is almost three orders of magnitude smaller than the central wavelength $(1.3 \mathrm{~mm})$ at that frequency and the filter begins to behave as a continuous gold film. While this transmission decrease can be offset with a larger $\varepsilon$, this increases the bandwidth of the pass band and noticeably reduces the transmission extinction at high frequencies. This reduced extinction can be seen in the various $\sigma$ samples in Figure 4(b). As $\sigma$ decreases, the ratio of bare substrate to unit cell increases (which, due to our scaled samples, mimics increasing $\varepsilon$ ), and these samples, subsequently, have vastly increased transmission at $30 \mathrm{THz}$.

To create a filter with a frequency higher than $8.51 \mathrm{THz}$, a smaller value of $\varepsilon$ is required. For the $\sigma=1(8.51 \mathrm{THz})$ filter, the geometry is completely limited by $\varepsilon$ and cannot be shrunk any further (for $\sigma=1, \mathrm{~W}=3 \times \varepsilon$ ). This could be counteracted with a smaller value of $\varepsilon$ using other fabrication methods, e.g., deep-UV photolithography or electron beam lithography with smaller achievable linewidths, but again, the design presented here was chosen for low cost, ease of fabrication, and widespread applicability.

Last, it is worth identifying the limits on the extinction range. The FTIR has shown high extinction up to $240 \mathrm{THz}$, but that is merely the limit of the measurement. The first practical limitation is the band gap of the HR-Si substrate at $271 \mathrm{THz}(1.11 \mu \mathrm{m} / 1.12 \mathrm{eV})$. While the band gap would continue to extinguish any transmitted spectrum, the photoexcitation of the substrate would also extinguish the desired pass band, defeating the purpose of the device. With a suitable substrate choice, such as a high band gap semiconductor or air, ${ }^{30}$ this limit could be pushed to even higher frequencies. On the low frequency side of the spectrum, the extinction is limited solely by the skin depth of the gold film.

In conclusion, we have shown that a metamaterial composite can have an ultra-broadband usable bandwidth that is suitable for virtually any $\mathrm{THz}$ source. We have constructed a series of bandpass filters that clearly demonstrate this concept, provided simple equations that can be used to construct filters at any frequency from $0.86-8.51 \mathrm{THz}$ without need for 
simulation or design, and described the nature of the scaling laws in the equations. These filters may be fabricated on both sides of the HR-Si substrate for further bandwidth reduction, and multiple filters may be used to narrow the transmitted spectrum even further as required. It is our hope that this work will bring an easy to fabricate, functional $\mathrm{THz}$ component to the laboratory, and expand the reach of metamaterial based $\mathrm{THz}$ components towards broadband functional components.

We acknowledge financial support from the Danish Council for Independent Research (FTP Projects HI-TERA and THz-COW) and the Carlsberg Foundation.

${ }^{1}$ J. B. Pendry, A. J. Holden, D. J. Robbins, and W. J. Stewart, IEEE Trans. Microwave Theory Tech. 47, 2075 (1999).

${ }^{2}$ D. Smith, W. J. Padilla, D. Vier, S. Nemat-Nasser, and S. Schultz, Phys. Rev. Lett. 84, 4184 (2000).

${ }^{3}$ J. B. Pendry, Phys. Rev. Lett. 85, 3966 (2000).

${ }^{4}$ A. Grbic and G. Eleftheriades, Phys. Rev. Lett. 92, 117403 (2004).

${ }^{5}$ N. B. Kundtz, D. R. Smith, and J. B. Pendry, Proc. IEEE 99, 1622 (2011).

${ }^{6}$ J. F. O'Hara, W. Withayachumnankul, and I. Al-Naib, J. Infrared, Millimeter, Terahertz Waves 33, 245 (2012).

${ }^{7}$ A. C. Strikwerda, K. Fan, H. Tao, D. V. Pilon, X. Zhang, and R. D. Averitt, Opt. Express 17, 136 (2008).

${ }^{8}$ N. K. Grady, J. E. Heyes, D. R. Chowdhury, Y. Zeng, M. T. Reiten, A. K. Azad, A. J. Taylor, D. A. R. Dalvit, and H.-T. Chen, Science 340, 1304 (2013).

${ }^{9}$ H.-T. Chen, J. F. O'Hara, A. K. Azad, and A. J. Taylor, Laser Photonics Rev. 5, 513 (2011).

${ }^{10}$ C. M. Watts, X. Liu, and W. J. Padilla, Adv. Mater. 24, OP98 (2012).

${ }^{11}$ H. Tao, E. A. Kadlec, A. C. Strikwerda, K. Fan, W. J. Padilla, R. D. Averitt, E. A. Shaner, and X. Zhang, Opt. Express 19, 21620 (2011).

${ }^{12}$ F. Alves, D. Grbovic, B. Kearney, N. V. Lavrik, and G. Karunasiri, Opt. Express 21, 13256 (2013).

${ }^{13}$ D. R. Smith, D. C. Vier, T. Koschny, and C. M. Soukoulis, Phys. Rev. E 71, 036617 (2005).

${ }^{14}$ D. H. Auston, K. P. Cheung, and P. R. Smith, Appl. Phys. Lett. 45, 284 (1984).
${ }^{15}$ H. Hirori, A. Doi, F. Blanchard, and K. Tanaka, Appl. Phys. Lett. 98, 091106 (2011).

${ }^{16}$ H. G. Roskos, M. D. Thomson, M. Kreß, and T. Löffler, Laser Photonics Rev. 1, 349 (2007).

${ }^{17}$ O. Paul, R. Beigang, and M. Rahm, Opt. Express 17, 18590 (2009).

${ }^{18}$ P. A. R. Ade, G. Pisano, C. Tucker, and S. Weaver, in Astron. Telesc. Instrum., edited by J. Zmuidzinas, W. S. Holland, S. Withington, and W. D. Duncan (International Society for Optics and Photonics, 2006), p. 62750U-1-62750U-15.

${ }^{19}$ R. Ulrich, Infrared Phys. 7, 37 (1967).

${ }^{20}$ H.-T. Chen, J. F. O'Hara, A. J. Taylor, R. D. Averitt, C. Highstrete, M. Lee, and W. J. Padilla, Opt. Express 15, 1084 (2007).

${ }^{21}$ CST, Microwave Studio, 2013.

${ }^{22}$ R. Malureanu, M. Zalkovskij, Z. Song, C. Gritti, A. Andryieuski, Q. He, L. Zhou, P. U. Jepsen, and A. V. Lavrinenko, Opt. Express 20, 22770 (2012).

${ }^{23}$ D. J. Cook and R. M. Hochstrasser, Opt. Lett. 25, 1210 (2000).

${ }^{24}$ N. Karpowicz, J. Dai, X. Lu, Y. Chen, M. Yamaguchi, H. Zhao, X.-C. Zhang, L. Zhang, C. Zhang, M. Price-Gallagher, C. Fletcher, O. Mamer, A. Lesimple, and K. Johnson, Appl. Phys. Lett. 92, 011131 (2008).

${ }^{25}$ P. Klarskov, A. C. Strikwerda, K. Iwaszczuk, and P. U. Jepsen, New J. Phys. 15, 075012 (2013).

${ }^{26}$ D. G. Cooke, F. C. Krebs, and P. U. Jepsen, Phys. Rev. Lett. 108, 056603 (2012).

${ }^{27}$ The fits used the simulated data from Table I and the Matlab Curvefitting Toolbox using a power law $f=A \sigma^{B}+C$. We have rounded to two decimal points in the text to match the precision of the values in Table I. The full results for $f_{0}$ are $\mathrm{A}=8.223$ (8.168, 8.278), $\mathrm{B}=-1.424(-1.449$, $-1.399), \mathrm{C}=0.2774(0.2191,0.3357) ; \Delta f_{1}$ are $\mathrm{A}=5.511(5.376,5.645)$; $\mathrm{B}=-1.557(-1.661,-1.454) ; \mathrm{C}=0.2178(0.07851,0.3571) ;$ and $\Delta f_{2}$ are $\mathrm{A}=3.161(2.972,3.35) ; \mathrm{B}=-1.046(-1.189,-0.9027) ; \mathrm{C}=-0.2098$ $(-0.4184,-0.001059)$, where the parantheses represent the $95 \%$ confidence bounds.

${ }^{28}$ W.-C. Chen, A. Totachawattana, K. Fan, J. L. Ponsetto, A. C. Strikwerda, X. Zhang, R. D. Averitt, and W. J. Padilla, Phys. Rev. B 85, 35112 (2012).

${ }^{29}$ O. Sydoruk, E. Tatartschuk, E. Shamonina, and L. Solymar, J. Appl. Phys. 105, 014903 (2009).

${ }^{30}$ M. Zalkovskij, R. Malureanu, C. Kremers, D. N. Chigrin, A. Novitsky, S. Zhukovsky, P. T. Tang, P. U. Jepsen, and A. V. Lavrinenko, Laser Photon. Rev. 7, 810 (2013).

${ }^{31}$ P. U. Jepsen and B. M. Fischer, Opt. Lett. 30, 29 (2005).

${ }^{32}$ C. S. Wang, J. M. Chen, R. Becker, and A. Zdetsis, Phys. Lett. A 44, 517 (1973). 